\title{
The Hahn-Banach Extension Theorem for Fuzzy Normed Spaces Revisited
}

\author{
Carmen Alegre and Salvador Romaguera \\ Instituto Universitario de Matemática Pura y Aplicada, Universitat Politècnica de València, Camí de Vera, \\ $s / n, 46022$ Valencia, Spain
}

Correspondence should be addressed to Salvador Romaguera; sromague@mat.upv.es

Received 13 March 2014; Accepted 13 May 2014; Published 28 May 2014

Academic Editor: Manuel Sanchis

Copyright (C) 2014 C. Alegre and S. Romaguera. This is an open access article distributed under the Creative Commons Attribution License, which permits unrestricted use, distribution, and reproduction in any medium, provided the original work is properly cited.

This paper deals with fuzzy normed spaces in the sense of Cheng and Mordeson. We characterize fuzzy norms in terms of ascending and separating families of seminorms and prove an extension theorem for continuous linear functionals on a fuzzy normed space. Our result generalizes the classical Hahn-Banach extension theorem for normed spaces.

\section{Introduction}

The Hahn-Banach extension theorem is without doubt one of the most important theorems in the whole theory of normed spaces. A classical formulation of such theorem is as follows.

Theorem 1. Let $(X,\|\cdot\|)$ be a normed space and let $f_{0}$ be a continuous linear functional on a subspace $X_{0}$ of $X$. There exists a continuous linear functional $f$ on $X$ such that $f_{\mid X_{0}}=f_{0}$ and $\left\|f_{0}\right\|^{*}=\|f\|^{*}$.

The fact that the class of normed spaces is strictly included in the class of fuzzy normed spaces motivates the following natural question: is it possible to give a theorem of HahnBanach type in the frame of fuzzy normed spaces which generalizes the classical one for normed spaces? In this paper, we will give an affirmative answer to this question by proving the following (the notation and terminology can be found along the paper).

Theorem 2. Let $(X, N, \wedge)$ be a fuzzy normed space such that the $\alpha$-seminorms corresponding to the fuzzy norm $(N, \wedge)$ are norms, and let $f_{0}$ be a continuous linear functional on a subspace $X_{0}$ of $X$. Then, there exists $\delta_{f_{0}} \in(0,1)$ for which the following two conditions are satisfied:
(1) for all $\alpha \in\left(0, \delta_{f_{0}}\right)$, there is a continuous linear functional $f_{\alpha}$ on $X$ such that $f_{\alpha \mid X_{0}}=f_{0}$ and $\left\|f_{\alpha}\right\|_{\alpha}^{*}=$ $\left\|f_{0}\right\|_{\alpha, X_{0}}^{*}$

(2) $N_{X_{0}}^{*}\left(f_{0}, t\right)=\sup \left\{N^{*}\left(f_{\alpha}, t\right): \alpha \in\left(0, \delta_{f_{0}}\right)\right\}$ for all $t>0$.

The antecedents of our study are in the paper by Bag and Samanta [1], where the authors obtain a theorem of HahnBanach type for a special class of fuzzy normed spaces, using in its proof the classical Hahn-Banach theorem for normed spaces. The scope of our result is a more general class of fuzzy normed spaces which allows us to deduce the classical theorem for normed spaces as a consequence.

It is well known that the fuzzy normed spaces are topological vector spaces, and hence the existence of continuous linear extension for each continuous linear functional defined on a linear subspace is guaranteed. Nevertheless, we do not use this fact in the proof of our result, but we give an explicit proof of the existence of a continuous linear extension because in this way we can also establish how to compute the fuzzy norm of the extension as stated in part (2) of Theorem 2 above. Some illustrative examples are also presented.

The first definition of fuzzy norm on a linear space was given by Katsaras [2] in 1984. Following this work, Felbin [3] 
offered in 1992 an alternative definition of a fuzzy norm on a linear space with an associated metric of Kaleva and Seikkala's type [4]. In 1994, Cheng and Mordeson [5] gave another definition of fuzzy norm that corresponds with the notion of a fuzzy metric as defined by Kramosil and Michálek in [6]. Bag and Samanta considered in [7] a fuzzy norm slightly different from this one and they proved a series of results that have been used in many subsequent works in this context. Although this definition is less restrictive than the one given by Cheng and Mordeson, the more interesting results given in the mentioned paper require the use of two very restrictive additional conditions which leave out of the scope of these results important examples of fuzzy normed spaces.

In this paper, we consider fuzzy normed spaces in the sense of Cheng and Mordeson. The organization of the paper is as follows. Section 2 comprises the basic notions on fuzzy normed spaces and some preliminary results. In Section 3, we study the relation between seminorms and fuzzy normed spaces. Theorems 8 and 12 of this section generalize the corresponding results given by Bag and Samanta in [7]. In this section, we also deduce from our approach the wellknown relationship between fuzzy normed spaces and locally convex spaces (see Theorem 14). Finally, in Section 4, we prove Theorem 2 above and deduce Theorem 1 from it.

\section{Terminology and Basic Notions}

According to $[8]$, a binary operation $*:[0,1] \times[0,1] \rightarrow$ $[0,1]$ is a continuous $t$-norm if $*$ satisfies the following conditions: (i) $*$ is associative and commutative; (ii) $*$ is continuous; (iii) $a * 1=a$ for every $a \in[0,1]$; (iv) $a * b \leq c * d$ whenever $a \leq c$ and $b \leq d$, with $a, b, c, d \in[0,1]$.

Three paradigmatic examples of continuous $t$-norms are $\wedge$, $\cdot$, and $*_{L}$ (the Lukasiewicz $t$-norm), which are defined by $a \wedge b=\min \{a, b\}, a \cdot b=a b$, and $a *_{L} b=\max \{a+b-1,0\}$, respectively. Recall that $*_{L} \leq \cdot \leq \wedge$. In fact, $* \leq \wedge$ for every continuous $t$-norm $*$.

Definition 3. If $X$ is a real vector space, a fuzzy norm on $X$ is a pair $(N, *)$ such that $*$ is a continuous $t$-norm and $N$ is a fuzzy set in $X \times[0, \infty)$ satisfying the following conditions for every $x, y \in X$, and $t, s \geq 0$ :

$$
\begin{aligned}
& (\mathrm{FN} 1) N(x, 0)=0 \\
& (\mathrm{FN} 2) N(x, t)=1 \text { for all } t>0 \Leftrightarrow x=\mathbf{0} \\
& (\mathrm{FN} 3) \\
& (\mathrm{FN} 4)
\end{aligned}
$$

The triple $(X, N, *)$ is called a fuzzy normed space.

If condition (FN5) is omitted we say that $(N, *)$ is a weak fuzzy norm on $X$, and the triple $(X, N, *)$ will be called a weak fuzzy normed space. These spaces will play a crucial role in the paper.

Recall that if in Definition 3 we put $*=\wedge$, then one has the notion of a fuzzy norm as given by Cheng and Morderson [5].
The class of fuzzy normed spaces is equivalent to a suitable subclass of Serstnev spaces in the case of continuous $t$-norms (see Remark 1 of [9]).

The following well-known example shows that every normed space can be considered as a fuzzy normed space.

Example 4. Let $(X,\|\cdot\|)$ be a normed space. Then,

(a) let $N: X \times[0, \infty) \rightarrow[0,1]$ given by $N(x, 0)=0$ for all $x \in X$ and let

$$
N(x, t)=\frac{t}{t+\|x\|},
$$

for all $x \in X$ and $t>0$. Then, $(N, *)$ is a fuzzy norm on $X$, where $*$ is any continuous $t$-norm. This fuzzy norm is called the standard fuzzy norm induced by $\|\cdot\|$.

(b) Let $N: X \times[0, \infty) \rightarrow[0,1]$ given by $N(x, t)=0$ if $t \leq$ $\|x\|$ and $N(x, t)=1$ if $t>\|x\|$. Then, $(N, *)$ is a fuzzy norm on $X$, where $*$ is any continuous $t$-norm. This fuzzy norm will be called the 01-fuzzy norm induced by $\|\cdot\|$.

If $(X, N, *)$ is a weak fuzzy normed space, the open ball $B_{N}(x, r, t)$ with center $x$, radius $r, 0<r<1$, and $t>0$ is defined as follows:

$$
B_{N}(x, r, t)=\{y \in X: N(y-x, t)>1-r\} .
$$

We note that $B_{N}(x, r, t)=x+B_{N}(\mathbf{0}, r, t)$, for all $x \in X$ and $0<r<1, t>0$. The closed ball $\bar{B}_{N}(x, r, t)$ with center $x$, radius $r, 0<r<1$, and $t>0$ is defined as follows:

$$
\bar{B}_{N}(x, r, t)=\{y \in X: N(y-x, t) \geq 1-r\} .
$$

It is clear that if $(X, N, *)$ is a weak fuzzy normed space, the fuzzy set $M_{N}$ in $X \times X \times[0, \infty)$ given by $M_{N}(x, y, t)=$ $N(y-x, t)$ is a fuzzy metric on $X$ in the sense of Kramosil and Michálek [6]. This fuzzy metric induces a topology $\tau_{N}$ on $X$, which has as a base the collection $\left\{B_{N}(x, r, t): x \in X, 0<\right.$ $r<1, t>0\}$. Moreover, $\tau_{N}$ is metrizable and the countable collection of balls $\left\{B_{N}(x, 1 / n, 1 / n): n=2,3, \ldots\right\}$ forms a fundamental system of neighborhoods of $x$, for all $x \in X$.

It is well known, and easy to see, that if $(X,\|\cdot\|)$ is a normed space, then the topology $\tau_{N}$ agrees with the topology induced by the norm $\|\cdot\|$, when $(N, *)$ is the standard fuzzy norm or the 01-fuzzy norm on $X$.

It is interesting to note that if $(X, N, \wedge)$ is a fuzzy normed space, the open (closed) balls are absorbent, balanced, and convex sets (Propositions 1 and 3 of [9]). However, if $(N, \wedge)$ is a weak fuzzy norm, it can happen that the open (closed) balls are not absorbent sets.

By omitting the condition of left continuity of the real function $N(x, \cdot)$ in the definition of fuzzy norm given by Cheng and Morderson [5], Bag and Samanta gave in [7] the following notion.

Definition 5 (see [7]). A BS-fuzzy norm on a real vector space $X$ is the pair $(N, \wedge)$ such that $N$ is a fuzzy set in $X \times \mathbb{R}$ satisfying the following conditions for every $x, y \in X$ and $t, s \in \mathbb{R}:$ 
(N1) $N(x, t)=0$, for all $t \leq 0$;

(N2) $N(x, t)=1$ for all $t>0 \Leftrightarrow x=\mathbf{0}$;

(N3) $N(c x, t)=N(x, t /|c|)$ for every $c \in \mathbb{R} \backslash\{0\}$;

(N4) $N(x+y, t+s) \geq N(x, t) \wedge N(y, s)$;

(N5) $N(x, \cdot)$ is nondecreasing and $\lim _{t \rightarrow \infty} N(x, t)=1$.

We point out that since (N2) and (N4) imply that $N(x, \cdot)$ is nondecreasing, this condition may be dropped from (N5).

A BS-fuzzy normed space satisfies the condition (N6) (page 691 of [7]) if $N(x, t)>0$, for all $t>0$, implies $x=\mathbf{0}$.

A BS-fuzzy normed space satisfies the condition (N7) (page 693 of [7]) if, for $x \neq \mathbf{0}, N(x, \cdot)$ is a continuous function of $\mathbb{R}$ which is strictly increasing on the subset $\{t: 0<$ $N(x, t)<1\}$.

The standard fuzzy norm and the 01-fuzzy norm induced by a norm are BS-fuzzy norms. The standard fuzzy norm satisfies (N7) but not (N6) while the 01-fuzzy norm satisfies (N6) but not (N7).

\section{Seminorms and Fuzzy Normed Spaces}

Bag and Samanta proved in Theorem 2.1 of [7] that if $(X, N, \wedge)$ is a BS-fuzzy normed space which satisfies condition (N6), then $\left\{\|\cdot\|_{\alpha}: \alpha \in(0,1)\right\}$ is an ascending family of norms on $X$, where

$$
\|x\|_{\alpha}=\inf \{t>0: N(x, t) \geq \alpha\} .
$$

From the proof of this result, it follows that if the condition (N6) is not required, then each $\|\cdot\|_{\alpha}$ is a seminorm on $X$.

In the next proposition, we give a shorter alternative proof of this result.

Proposition 6. Let $(X, N, \wedge)$ be a fuzzy normed space and let $\alpha \in(0,1)$. Then, the following hold.

(a) The function $\|\cdot\|_{\alpha}: X \rightarrow[0, \infty)$ given by

$$
\|x\|_{\alpha}=\inf \{t>0: N(x, t) \geq \alpha\}
$$

is a seminorm on X. In fact, it is the Minkowski functional of the ball $\bar{B}(0,1-\alpha, 1)$.

(b) The family $\left\{\|\cdot\|_{\alpha}: \alpha \in(0,1)\right\}$ is separating.

Proof. (a) Let $p$ be the Minkowski functional of the closed ball $\bar{B}_{N}(\mathbf{0}, 1-\alpha, 1)$. Clearly, $p$ is a seminorm because $\bar{B}_{N}(\mathbf{0}, 1-$ $\alpha, 1)$ is an absolutely convex absorbent set. Then,

$$
\begin{aligned}
p(x) & =\inf \left\{\lambda>0: x \in \lambda \bar{B}_{N}(0,1-\alpha, 1)\right\} \\
& =\inf \left\{\lambda>0: N\left(\frac{x}{\lambda}, 1\right) \geq \alpha\right\} \\
& =\inf \{\lambda>0: N(x, \lambda) \geq \alpha\}=\|x\|_{\alpha} .
\end{aligned}
$$

(b) If $\|x\|_{\alpha}=0$ for every $\alpha \in(0,1)$, then $N(x, t) \geq 1-1 / n$, for every $n \in \mathbb{N}$ and $t>0$. Therefore, $N(x, t)=1$ for all $t>0$ and so $x=\mathbf{0}$.
Example 7. (a) Let $(X,\|\cdot\|)$ be a normed space and let $(N, \wedge)$ be the standard fuzzy norm induced by $\|\cdot\|$. Then, $\|x\|_{\alpha}=$ $(\alpha /(1-\alpha))\|x\|$. Note that $(N, \wedge)$ does not satisfy condition (N6) but $\|\cdot\|_{\alpha}$ is a norm for all $\alpha \in(0,1)$.

(b) Let $(\mathrm{X},\|\cdot\|)$ be a normed space and let $(N, \wedge)$ be the $01-$ fuzzy norm induced by $\|\cdot\|$. Then, $\|x\|_{\alpha}=\|x\|$, for all $\alpha \in(0,1)$.

If $(X, N, \wedge)$ is a fuzzy normed space, the family $\left\{\|\cdot\|_{\alpha}\right.$ : $\alpha \in(0,1)\}$ will be called the $\alpha$-seminorms corresponding to the fuzzy norm $(N, \wedge)$.

Bag and Samanta stated in Theorem 2.2 of [7] that given an ascending family $\left\{\|\cdot\|_{\alpha}: \alpha \in\right] 0,1[\}$ of norms on a real linear space $X$, then the pair $(X, \wedge)$ is a BS-fuzzy norm on $X$, where $N: X \times[0, \infty) \rightarrow[0,1]$ is defined by $N(\mathbf{0}, 0)=0$, and $N(x, t)=\sup \left\{\alpha \in(0,1):\|x\|_{\alpha} \leq t\right\}$, otherwise.

Next, we generalize this result for the case that $\left\{\|\cdot\|_{\alpha}\right.$ : $\alpha \in(0,1)\}$ is a family of extended separating seminorms on $X$. In fact, our extension requires an explicit proof because Bag-Samanta's construction of $N$ presents some slight disarrangement. Indeed, if $(X,\|\cdot\|)$ is a nontrivial normed space and put $\|\cdot\|_{\alpha}=\|\cdot\|$ for all $\alpha \in(0,1)$, then for each $x \in X \backslash\{\boldsymbol{0}\}$ it follows that $N(x, t)$ is not defined whenever $\|x\|>t$.

Theorem 8. Let $\left\{\|\cdot\|_{\alpha}: \alpha \in(0,1)\right\}$ be an ascending family of separating seminorms on a real linear space $X$, and let $\|\cdot\|_{0}$ be given by $\|x\|_{0}=0$, for all $x \in X$. Then, the pair $(N, \wedge)$ is a fuzzy norm on $X$, where $N: X \times[0, \infty) \rightarrow[0,1]$ is given by $N(x, 0)=0$, for all $x \in X$, and

$$
N(x, t)=\sup \left\{\alpha \in[0,1):\|x\|_{\alpha}<t\right\}
$$

for all $x \in X$ and $t>0 .(N, \wedge)$ will be called the fuzzy norm induced by the seminorms $\left\{\|\cdot\|_{\alpha}: \alpha \in(0,1)\right\}$.

Proof. (FN1) Consider that $N(x, 0)=0$, by definition of $N$.

(FN2) If $N(x, t)=1$ for all $t>0$, then $\|x\|_{\alpha}<t$ for all $\alpha \in[0,1)$. Therefore, $\|x\|_{\alpha}=0$ for all $\alpha \in[0,1)$. Consequently, $x=\mathbf{0}$, since the family of seminorms is separating.

(FN3) Let $c \in \mathbb{R} \backslash\{0\}, x \in X$, and $t>0$. Then,

$$
\begin{aligned}
N(c x, t) & =\sup \left\{\alpha \in[0,1):\|c x\|_{\alpha}<t\right\} \\
& =\sup \left\{\alpha \in[0,1):\|x\|_{\alpha}<\frac{t}{|c|}\right\} \\
& =N\left(x, \frac{t}{|c|}\right) .
\end{aligned}
$$

(FN4) Let $x, y \in X$ and let $s, t \geq 0$. Suppose that $N(x, t) \leq$ $N(y, s)$. Then, $\|y\|_{\alpha}<s$ whenever $\|x\|_{\alpha}<t$. Since $\|x+y\|_{\alpha} \leq$ $\|x\|_{\alpha}+\|y\|_{\alpha}$, it follows that $\|x+y\|_{\alpha}<t+s$ whenever $\|x\|_{\alpha}<t$ for all $\alpha \in[0,1[$. Consequently, $N(x+y, t+s) \geq N(x, t)=$ $N(x, t) \wedge N(y, s)$.

(FN5) Let $x \in X$ and let $\varepsilon>0$. There exits $\alpha_{0} \in(0,1)$ such that $1-\alpha_{0}<\varepsilon$. Let $t$ be such that $t>\|x\|_{\alpha_{0}}$. Then, $N(x, t) \geq \alpha_{0}>1-\varepsilon$. Hence, $\lim _{t \rightarrow \infty} N(x, t)=1$.

(FN6) Let $x \in X$ and let $t_{0}>0$.

If $N\left(x, t_{0}\right)=0$, then $N(x, t)=N\left(x, t_{0}\right)=0$, for all $t<t_{0}$. 
Suppose $0<N\left(x, t_{0}\right) \leq 1$. Then, given $\varepsilon>0$, there is $\alpha_{0} \in(0,1)$ such that $\|x\|_{\alpha_{0}}<t_{0}$ and $N\left(x, t_{0}\right)-\alpha_{0}<\varepsilon$. Let $t$ be such that $\|x\|_{\alpha_{0}}<t<t_{0}$. Then, $N(x, t) \geq \alpha_{0}$ and so

$$
N\left(x, t_{0}\right)-N(x, t) \leq N\left(x, t_{0}\right)-\alpha_{0}<\varepsilon .
$$

Therefore, $N(x, \cdot)$ is left continuous.

Remark 9. If in the above theorem we define $N(x, t)=$ $\sup \left\{\alpha \in[0,1):\|x\|_{\alpha} \leq t\right\}$ instead of $N(x, t)=\sup \{\alpha \in[0,1[$ : $\left.\|x\|_{\alpha}<t\right\}$, the left continuity of $N(x, \cdot)$ is not guaranteed. Indeed, if $(X,\|\cdot\|)$ is a nontrivial normed space and put $\|\cdot\|_{\alpha}=\|\cdot\|$ for all $\alpha \in(0,1)$, then $N(x, t)=0$ if $t<\|x\|$ and $N(x, t)=1$ if $t \geq\|x\|$, and this function is not left continuous at $t=\|x\|$ for every $x \neq \mathbf{0}$.

Let $X$ be a linear space and let $p: X \rightarrow \mathbb{R}^{+} \cup\{\infty\}$. If $p$ satisfies the conditions of a seminorm, we say that $p$ is an extended seminorm.

If $\left\{p_{i}: i \in I\right\}$ is a family of extended seminorms on $X$, we say that the family is separating if for all $x \in X, x \neq 0$, there are $j, k \in I$ such that $p_{j}(x) \neq 0$ and $p_{k}(x) \neq \infty$.

If we consider an ascending family of separating extended seminorms on $X$, mimicking the proof of Theorem 8 , we obtain the following result.

Theorem 10. Let $\left\{\|\cdot\|_{\alpha}: \alpha \in(0,1)\right\}$ be an ascending family of separating extended seminorms on a real linear space $X$, and let $\|\cdot\|_{0}$ be given by $\|x\|_{0}=0$, for all $x \in X$. Then, the pair $(N, \wedge)$ is a weak fuzzy norm on $X$, where $N: X \times[0, \infty) \rightarrow[0,1]$ is given by $N(x, 0)=0$, for all $x \in X$, and

$$
N(x, t)=\sup \left\{\alpha \in[0,1):\|x\|_{\alpha}<t\right\}
$$

for all $x \in X$ and $t>0$. $(N, \wedge)$ will be called the weak fuzzy norm induced by the seminorms $\left\{\|\cdot\|_{\alpha}: \alpha \in(0,1)\right\}$.

Bag and Samanta stated in Theorem 2.3 of [7] that if $(X, N, \wedge)$ is a BS-fuzzy normed space which satisfies (N6) and (N7), then $N$ coincides with the BS-fuzzy norm induced by the $\alpha$-norms corresponding to the fuzzy norm $(N, \wedge)$. Next, we generalize this theorem to the case that $(X, N, \wedge)$ is a fuzzy normed space. To prove this result, first we prove the following lemma.

Lemma 11. Let $(X, N, \wedge)$ be a fuzzy normed space. Let $\left\{\|\cdot\|_{\alpha}\right.$ : $\alpha \in(0,1)\}$ be the $\alpha$-seminorms corresponding to the fuzzy $\operatorname{norm}(N, \wedge)$.

(a) If $\|x\|_{\alpha}<t$, then $N(x, t) \geq \alpha$.

(b) If $N(x, t)>\alpha$, then $\|x\|_{\alpha}<t$.

Proof. (a) Suppose that $N(x, t)<\alpha$. Since $N(x, \cdot)$ is nondecreasing, it follows that $t \leq s$ for every $s>0$ such that $N(x, s) \geq \alpha$. Therefore,

$$
t \leq \inf \{s>0: N(x, s) \geq \alpha\}=\|x\|_{\alpha}
$$

which provides a contradiction because $\|x\|_{\alpha}<t$.

(b) If $N(x, t)>\alpha$, then $\|x\|_{\alpha} \leq t$, by definition of $\|\cdot\|_{\alpha}$. Suppose that $\|x\|_{\alpha}=t$. Since $N(x, \cdot)$ is left continuous and
$N(x, t)>\alpha$, there exists $t^{\prime}<t$ such that $N\left(x, t^{\prime}\right) \geq \alpha$. Now, if $N\left(x, t^{\prime}\right) \geq \alpha$, then $t^{\prime} \geq\|x\|_{\alpha}=t$ which provides a contradiction.

Theorem 12. Let $(X, N, \wedge)$ be a fuzzy normed space. Let $\left\{\|\cdot\|_{\alpha}: \alpha \in(0,1)\right\}$ be the $\alpha$-seminorms corresponding to the fuzzy norm $(N, \wedge)$. If $\left(N^{\prime}, \wedge\right)$ is the fuzzy norm induced by the seminorms $\left\{\|\cdot\|_{\alpha}: \alpha \in(0,1)\right\}$, then $N=N^{\prime}$.

Proof. Let $x \in X$ and let $t>0$. Let $\alpha \in[0,1)$ such that $\|x\|_{\alpha}<$ $t$. Then, by Lemma 11 (a), $N(x, t) \geq \alpha$. Now, if $N(x, t) \geq \alpha$ for every $\alpha \in[0,1)$ such that $\|x\|_{\alpha}<t$, then

$$
N(x, t) \geq \sup \left\{\alpha \in[0,1):\|x\|_{\alpha}<t\right\}=N^{\prime}(x, t) .
$$

Suppose that there exist $x \in X$ and $t>0$ such that $N(x, t)>$ $N^{\prime}(x, t)$. Let $\beta \in(0,1)$ such that $N(x, t)>\beta>N^{\prime}(x, t)$. By Lemma 11 (b), $\|x\|_{\beta}<t$, so $N^{\prime}(x, t) \geq \beta$, which provides a contradiction.

By using known properties of locally convex vector spaces, the family of $\alpha$-seminorms corresponding to the fuzzy norm $(N, \wedge)$ induces a topology $\tau$ on $X$ such that $(X, \tau)$ is a Hausdorff locally convex space. Since the family of $\alpha$ seminorms is ascending, a base of neighborhoods of the origin in this topology consists of the sets of the form

$$
U(\alpha, \varepsilon)=\left\{x \in X:\|x\|_{\alpha}<\varepsilon\right\}, \quad \varepsilon>0, \alpha \in(0,1) .
$$

Proposition 13. Let $(X, N, \wedge)$ be a fuzzy normed space. Let $\tau_{N}$ be the topology induced on $X$ by the fuzzy norm $(N, \wedge)$ and let $\tau$ be the topology induced on $X$ by the family of $\alpha$-seminorms corresponding to the fuzzy norm $(N, \wedge)$. Then, $\tau_{N}=\tau$.

Proof. Take the open ball $B_{N}(\mathbf{0}, r, t)$ and let it be with $0<$ $r<1$ and $t>0$. Then, $U(1-r / 2, t) \subseteq B_{N}(\mathbf{0}, r, t)$ because if $\|x\|_{1-r / 2}<t$, then, by Lemma 11 (a), $N(x, t) \geq 1-r / 2>1-r$. Consequently, $\tau_{N}$ is coarser than $\tau$.

Now, given $U(\alpha, \varepsilon)$ with $\varepsilon>0$ and $\alpha \in(0,1)$ then, by Lemma 11 (b), $B_{N}(\mathbf{0}, 1-\alpha, \varepsilon) \subseteq U(\alpha, \varepsilon)$. Therefore, $\tau$ is coarser than $\tau_{N}$.

As a consequence of the above results, it is possible to obtain the relationship between fuzzy normed spaces and locally convex spaces. This result was obtained by Radu [10] in the realm of random normed spaces of Serstnev [11]. A timely update of this fact was presented in [9].

Theorem 14 (see $[9,10])$. (a) Let $(X, N, \wedge)$ be a fuzzy normed space. Then, $\left(X, \tau_{N}\right)$ is a metrizable locally convex space.

(b) Let $(X, \tau)$ be a metrizable locally convex space. Then, there is a fuzzy norm $(N, \wedge)$ on $X$ such that $\tau_{N}=\tau$.

Proof. (a) It is immediate by Proposition 13.

(b) If $(X, \tau)$ is a metrizable locally convex space, $\tau$ is determined by a separating family of seminorms $\left\{p_{i}: i \in \mathbb{N}\right\}$ on $X$. Let $\left\{q_{i}: i \in \mathbb{N}\right\}$ be the ascending family of seminorms such that $q_{i}=\max \left\{p_{1}, \ldots, p_{i}\right\}$, and then $\tau$ is also determined by this family, and the sets

$$
V\left(q_{i}, \varepsilon\right)=\left\{x \in X: q_{i}(x)<\varepsilon\right\}, \quad \varepsilon>0, i \in \mathbb{N},
$$

form a base of neighborhoods of the origin in $(X, \tau)$. 
Let $\left\{\|\cdot\|_{\alpha}: \alpha \in(0,1)\right\}$ be the ascending family of separating seminorms such that $\|x\|_{\alpha}=q_{n}(x)$ if $\alpha \in((n-$ 1) $/ n, n /(n+1)]$, for all $n \in \mathbb{N}$. By Theorem 8 , this family induces a fuzzy norm $(N, \wedge)$ on $X$. Next, we show that $\tau_{N}=\tau$. To this end, take the open ball $B_{N}(\mathbf{0}, 1 / n, 1 / n)$ with $n \geq 2$. We claim that $V\left(q_{n}, 1 / n\right) \subseteq B_{N}(0,1 / n, 1 / n)$. Indeed, if $q_{n}(x)<$ $1 / n$, then $\|x\|_{\alpha}<1 / n$ for all $\alpha \in(0, n /(n+1)]$. Therefore,

$$
\begin{aligned}
N\left(x, \frac{1}{n}\right) & =\sup \left\{\alpha \in[0,1):\|x\|_{\alpha}<\frac{1}{n}\right\} \geq \frac{n}{n+1} \\
& =1-\frac{1}{n+1}>1-\frac{1}{n} .
\end{aligned}
$$

On the other hand, if we take $V\left(q_{n}, \varepsilon\right), n \geq 1, \varepsilon>0$, then $B_{N}(\mathbf{0}, 1 /(n+1), \varepsilon) \subseteq V\left(q_{n}, \varepsilon\right)$. Indeed, if $N(x, \varepsilon)>1-1 /(n+1)$, then $\|x\|_{n /(n+1)}<\varepsilon$, and hence, $q_{n}(x)<\varepsilon$.

Remark 15. If $(X, N, \wedge)$ is a weak fuzzy normed space, then $\left(X, \tau_{N}\right)$ is a metrizable topological space, but it is not a topological vector space since the open (closed) balls are not absorbent sets, in general.

The following example shows that the family of $\alpha$ seminorms corresponding to a fuzzy norm $(N, \wedge)$ compatible with a nonnormable metrizable locally convex space can be a family of norms.

Example 16. Let $A=\left(a_{n, k}\right)_{n, k \in \mathbb{N}}$ be a matrix of nonnegative real numbers such that $0<a_{n, k} \leq a_{n, k+1}$ for all $n, k \in \mathbb{N}$. Let $p \in[1, \infty)$ and let $\lambda_{p}(A)$ be the Köthe echelon space of order $p$,

$$
\begin{gathered}
\lambda_{p}(A)=\left\{x \in \mathbb{R}^{\mathbb{N}}: q_{n}(x)=\left(\sum_{k=1}^{\infty} a_{n, k}\left|x_{k}\right|^{p}\right)^{1 / p}<\infty\right. \\
\text { for all } n \in \mathbb{N}\} .
\end{gathered}
$$

It is well known (Lemma 27.1 of [12]) that $\lambda_{p}(A)$ is a locally convex space for the topology $\tau$ generated by the seminorms $\left\{q_{n}: n \in \mathbb{N}\right\}$ and that $\tau$ has a compatible complete metric. Since $a_{n, k}>0$ for all $n, k \in \mathbb{N}$, then $q_{n}$ is a norm for all $n \in \mathbb{N}$.

By Theorem $14(\mathrm{~b})$, there is a fuzzy norm $(N, \wedge)$ on $\lambda_{p}(A)$ such that $\tau_{N}=\tau$. Moreover, the proof of Theorem 14 (b) shows that $N$ is induced by the ascending family of norms $\left\{\|\cdot\|_{\alpha}: \alpha \in(0,1)\right\}$ given by $\|x\|_{\alpha}=q_{n}(x)$, where $\alpha \in((n-1) / n, n /(n+1)], n \in \mathbb{N}$. Finally, if we consider the fuzzy normed space $\left(\lambda_{p}(A), N, \wedge\right)$, the $\alpha$-seminorms corresponding to the fuzzy norm $(N, \wedge)$ are the norms $\left\{\|\cdot\|_{\alpha}\right.$ : $\alpha \in(0,1)\}$, by Theorem 12 .

\section{Continuous Linear Functionals and the Hahn-Banach Theorem}

Let $(X, N, \wedge)$ be a fuzzy normed space and let $\left(N_{s}, \wedge\right)$ be the standard fuzzy norm on $\mathbb{R}$, that is; $N_{s}(x, 0)=0$ for $x \in \mathbb{R}$ and
$N_{s}(x, t)=t /(t+|x|)$ for all $x \in \mathbb{R}$ and $t>0$ (see Example 4 (a)).

Denote by $X^{*}$ the set of all continuous linear mappings from $\left(X, \tau_{N}\right)$ to $\left(\mathbb{R}, \tau_{N_{s}}\right)$. (Note that $\tau_{N_{s}}$ is the usual topology of $\mathbb{R}$.)

Proposition 17. Let $(X, N, \wedge)$ be a fuzzy normed space and let $\left\{\|\cdot\|_{\alpha}: \alpha \in(0,1)\right\}$ be the $\alpha$-seminorms corresponding to the fuzzy norm $(N, \wedge)$. Then, $f \in X^{*}$ if and only if there exist $\alpha \in(0,1)$ and $M>0$ such that $|f(x)| \leq M\|x\|_{\alpha}$ for every $x \in X$.

Proof. Let $f \in X^{*}$. By Proposition 13, there are $\alpha \in(0,1)$ and $\varepsilon>0$ such that $f(U(\alpha, \varepsilon)) \subseteq[-1,1]$, where $U(\alpha, \varepsilon)=\{x \in X$ : $\left.\|x\|_{\alpha}<\varepsilon\right\}$. If $x \in X$ and $\|x\|_{\alpha} \neq 0$, then $\varepsilon x /\|x\|_{\alpha} \in U(\alpha, \varepsilon)$ and so

$$
|f(x)| \leq \frac{1}{\varepsilon}\|x\|_{\alpha} .
$$

If $\|x\|_{\alpha}=0$, then $n x \in U(\alpha, \varepsilon)$ for all $n \in \mathbb{N}$. Therefore, $|f(x)| \leq 1 / n$ for each $n \in \mathbb{N}$ and then $|f(x)|=0$. Consequently,

$$
|f(x)|=\frac{1}{\varepsilon}\|x\|_{\alpha}=0 .
$$

Conversely, suppose there exist $\alpha \in(0,1)$ and $M>0$ such that $|f(x)| \leq M\|x\|_{\alpha}$ for every $x \in X$. Then, given $\varepsilon>0$, we have that $f(U(\alpha, \varepsilon / M)) \subseteq[-\varepsilon, \varepsilon]$. Since $U(\alpha, \varepsilon / M)$ is a neighborhood of the origin in the topology $\tau_{N}$, we have that $f$ is continuous at the origin and, by linearity, $f$ is continuous at each point of $X$.

For each $f \in X^{*}$ define $\|f\|_{0}^{*}=0$ and

$$
\|f\|_{\alpha}^{*}=\sup \left\{|f(x)|:\|x\|_{1-\alpha} \leq 1\right\},
$$

whenever $\alpha \in(0,1)$.

Proposition 18. Let $(X, N, \wedge)$ be a fuzzy normed space. Then, $\left\{\|\cdot\|_{\alpha}^{*}: \alpha \in(0,1)\right\}$ is an ascending family of extended norms on $X^{*}$.

Proof. It is easy to show that $\|\cdot\|_{\alpha}^{*}$ is an extended seminorm on $X^{*}$ for each $\alpha \in(0,1)$. Now, if $\|f\|_{\alpha}^{*}=0$ for all $\alpha \in(0,1)$, then $|f(x)|=0$ for all $x \in U(1-\alpha, 1)$, where $U(1-\alpha, 1)=$ $\left\{x \in X:\|x\|_{1-\alpha} \leq 1\right\}$. Since $U(1-\alpha, 1)$ is absorbent, given $x \in X$, there exists $k>0$ such that $k x \in U(1-\alpha, 1)$. Then, $f(k x)=k f(x)=0$ and so $f(x)=0$.

If $\alpha \leq \beta$, then $1-\beta \leq 1-\alpha$ and then $\|f\|_{\alpha}^{*} \leq\|f\|_{\beta}^{*}$. On the other hand, by Proposition 17, for every $f \in X^{*}$, there is $\alpha \in(0,1)$ such that $\|f\|_{\alpha}^{*}<\infty$. Therefore, $\left\{\|\cdot\|_{\alpha}^{*}: \alpha \in(0,1)\right\}$ is an ascending family of extended norms on $X^{*}$.

The following example shows that $\|f\|_{\alpha}^{*}$ can be infinity.

Example 19. Let $X$ be the linear space of all sequences $x:=$ $\left(x_{n}\right)_{n}$ of real scalars. $X$ is a metrizable locally convex vector space for the topology $\tau$ generated by the seminorms $p_{n}(x)=$ $\left|x_{n}\right|, n \in \mathbb{N}$. 
By Theorem 14 (b), there is a fuzzy norm $(N, \wedge)$ on $X$ such that $\tau_{N}=\tau$. Moreover, if $q_{i}=\max \left\{p_{1}, \ldots, p_{i}\right\}$, following the proof of this result, $N$ is induced by the ascending family of separating seminorms $\left\{\|\cdot\|_{\alpha}: \alpha \in(0,1)\right\}$ given by $\|x\|_{\alpha}=$ $q_{n}(x)$ if $\alpha \in((n-1) / n, n /(n+1)]$, for all $n \in \mathbb{N}$.

By Theorem 12, this family of seminorms is the $\alpha$ seminorms corresponding to the fuzzy norm $(N, \wedge)$.

Let $f: X \rightarrow \mathbb{R}$ be the linear function given by $f(x)=$ $x_{1}+x_{2}+x_{3}$. Since $|f(x)| \leq 3 q_{3}(x)=3\|x\|_{3 / 4}$, we have that $f$ is continuous and $\|f\|_{1 / 4}^{*} \leq 3$.

For each $k \in \mathbb{N}$, let $x^{k}$ be the sequence given by $x_{i}^{k}=0$ if $i \neq 3$ and $x_{3}^{k}=k$. Since $\left\|x^{k}\right\|_{1 / 2}=q_{2}\left(x^{k}\right)=0$ and $\left|f\left(x^{k}\right)\right|=k$, for all $k \in \mathbb{N}$, we have

$$
\|f\|_{1 / 2}^{*}=\sup \left\{|f(x)|:\|x\|_{1-1 / 2} \leq 1\right\} \geq k,
$$

for all $k \in \mathbb{N}$. Therefore, $\|f\|_{1 / 2}^{*}=\infty$ and $1 / 4 \leq \delta_{f} \leq 1 / 2$, where $\delta_{f}=\sup \left\{\alpha \in(0,1):\|f\|_{\alpha}^{*}<\infty\right\}$.

By Theorem 10 and Proposition 18, the pair $\left(N^{*}, \wedge\right)$ is a weak fuzzy norm on $X^{*}$, where $N^{*}(f, 0)=0$ for all $f \in X^{*}$, and

$$
N^{*}(f, t)=\sup \left\{\alpha \in[0,1):\|f\|_{\alpha}^{*}<t\right\},
$$

for all $f \in X^{*}$.

Lemma 20. Let $(X, N, \wedge)$ be a fuzzy normed space. If $f \in X^{*}$ and $t>0$, then

$$
N^{*}(f, t)=\sup \left\{\alpha \in\left[0, \delta_{f}\right):\|f\|_{\alpha}^{*}<t\right\},
$$

where $\delta_{f}=\sup \left\{\alpha \in(0,1):\|f\|_{\alpha}^{*}<\infty\right\}$.

Proof. If $\|f\|_{\delta_{f}}^{*}<t$, since $\|f\|_{\alpha}^{*}=\infty$, for all $\alpha>\delta_{f}$, we have

$$
\begin{aligned}
\sup & \left\{\alpha \in[0,1):\|f\|_{\alpha}^{*}<t\right\} \\
& =\sup \left\{\alpha \in\left[0, \delta_{f}\right):\|f\|_{\alpha}^{*}<t\right\}=\delta_{f} .
\end{aligned}
$$

If $\|f\|_{\delta_{f}}^{*} \geq t$, then $\|f\|_{\alpha}^{*} \geq t$, for all $\alpha \geq \delta_{f}$. Hence,

$$
\sup \left\{\alpha \in[0,1):\|f\|_{\alpha}^{*}<t\right\}=\sup \left\{\alpha \in\left[0, \delta_{f}\right):\|f\|_{\alpha}^{*}<t\right\} .
$$

In order to prove Theorem 2, we will also use the following terminology.

If $X_{0}$ is a linear subspace of a fuzzy normed space $(X, N, \wedge)$, we denote by $X_{0}^{*}$ the set of all continuous linear mappings from $\left(X_{0}, \tau_{N \mid X_{0}}\right)$ to $\left(\mathbb{R}, \tau_{N_{s}}\right)$. If $f \in X_{0}^{*}$ and $\alpha \in$ $(0,1)$, we denote by $\|\cdot\|_{\alpha, X_{0}}^{*}$ the extended norm on $X_{0}$ given by

$$
\|f\|_{\alpha, X_{0}}^{*}=\sup \left\{|f(x)|: x \in X_{0},\|x\|_{1-\alpha} \leq 1\right\} .
$$

By $\left(N_{X_{0}}^{*}, \wedge\right)$, we will denote the weak fuzzy norm on $X_{0}^{*}$ given by $N_{X_{0}}^{*}(f, 0)=0$ and

$$
N_{X_{0}}^{*}(f, t)=\sup \left\{\alpha \in[0,1):\|f\|_{\alpha, X_{0}}^{*}<t\right\},
$$

for all $f \in X_{0}^{*}$ and $t>0$.
Lemma 21 (Theorem 5, p. 132 of [13]). Let $p$ be a convex functional on a real linear space $X$ and let $f_{0}$ be a linear functional on a subspace $X_{0}$ of $X$ such that $f_{0}(x) \leq p(x)$ for all $x \in X_{0}$. Then, there is a linear functional $f$ on $X$ such that $f_{\mid X_{0}}=f_{0}$ and $f(x) \leq p(x)$ for all $x \in X$.

Proof of Theorem 2. Since $f_{0} \in X_{0}^{*}$, by Proposition 17, there exists $\alpha \in(0,1)$ such that $\left\|f_{0}\right\|_{\alpha, X_{0}}^{*}<+\infty$. Let $\delta_{f_{0}}=\sup \{\alpha \epsilon$ $\left.(0,1):\left\|f_{0}\right\|_{\alpha, X_{0}}^{*}<\infty\right\}$. If $\alpha \in\left(0, \delta_{f_{0}}\right)$, we define $p_{\alpha}: X \rightarrow$ $[0, \infty)$ by $p_{\alpha}(x)=\left\|f_{0}\right\|_{\alpha, X_{0}}^{*}\|x\|_{1-\alpha}$ for all $x \in X$. Clearly, $p_{\alpha}$ is a convex functional on $X_{0}$.

Let $x \in X_{0} \backslash\{\mathbf{0}\}$. Since $f_{0}\left(x /\|x\|_{1-\alpha}\right) \leq\left\|f_{0}\right\|_{\alpha, X_{0}}^{*}$, it follows that $f_{0}(x) \leq p_{\alpha}(x)$ for all $x \in X_{0}$ and $\alpha \in\left(0, \delta_{f_{0}}\right)$. Therefore, by Lemma 21, for each $\alpha \in\left(0, \delta_{f_{0}}\right)$, there is a linear functional $f_{\alpha}$ on $X$ such that $f_{\alpha \mid X_{0}}=f_{0}$ and

$$
f_{\alpha}(x) \leq p_{\alpha}(x)=\left\|f_{0}\right\|_{\alpha, X_{0}}^{*}\|x\|_{1-\alpha},
$$

for all $x \in X$. By Proposition 17, $f_{\alpha}$ is continuous on $X$ for all $\alpha \in\left(0, \delta_{f_{0}}\right)$. Moreover, since $\left|f_{\alpha}(x)\right| \leq\left\|f_{0}\right\|_{\alpha, X_{0}}^{*}$ for every $x \in X$ such that $\|x\|_{1-\alpha} \leq 1$, we obtain that $\left\|f_{\alpha}\right\|_{\alpha}^{*}=\left\|f_{0}\right\|_{\alpha, X_{0}}^{*}$.

Next, we show that $N_{X_{0}}^{*}\left(f_{0}, t\right)=\sup \left\{N^{*}\left(f_{\alpha}, t\right): \alpha \epsilon\right.$ $\left.\left(0, \delta_{f_{0}}\right)\right\}$ for all $t>0$.

By definition of $N_{X_{0}}^{*}$, it immediately follows that $N_{X_{0}}^{*}\left(f_{0}, t\right) \geq N^{*}\left(f_{\alpha}, t\right)$, for all $\alpha \in\left(0, \delta_{f_{0}}\right)$. Hence,

$$
N_{X_{0}}^{*}\left(f_{0}, t\right) \geq \sup \left\{N^{*}\left(f_{\alpha}, t\right): \alpha \in\left(0, \delta_{f_{0}}\right)\right\} .
$$

On the other hand, if $\alpha \in\left(0, \delta_{f_{0}}\right)$ and $\left\|f_{0}\right\|_{\alpha, X_{0}}^{*}<t$, we have that $\left\|f_{\alpha}\right\|_{\alpha}^{*}<t$ and then $N^{*}\left(f_{\alpha}, t\right) \geq \alpha$. Therefore,

$$
\begin{aligned}
& \sup \left\{N^{*}\left(f_{\alpha}, t\right): \alpha \in\left(0, \delta_{f_{0}}\right)\right\} \\
& \geq \sup \left\{\alpha \in\left[0, \delta_{f}\right):\left\|f_{0}\right\|_{\alpha, X_{0}}^{*}<t\right\} \\
&=N_{X_{0}}^{*}\left(f_{0}, t\right) .
\end{aligned}
$$

As we indicated in Section 1, the classical Hahn-Banach theorem for normed spaces (Theorem 1) can be obtained from Theorem 2.

Indeed, let $(X,\|\cdot\|)$ be a normed space and let $f_{0}$ be a continuous linear functional on a subspace $X_{0}$ of $X$. Let $(N, \wedge)$ be the 01 -fuzzy norm on $X$ induced by $(X,\|\cdot\|)$. The $\alpha$-seminorms corresponding to $(N, \wedge)$ are $\|\cdot\|_{\alpha}=\|\cdot\|$ for every $\alpha \in(0,1)$. Moreover, $\|\cdot\|_{\alpha}^{*}=\|\cdot\|^{*}$ for every $\alpha \in(0,1)$ and $\delta_{g}=1$ for all $g \in X^{*}$. Therefore, by applying Theorem 2, we have that for each $\alpha \in(0,1)$ there is $f_{\alpha} \in X^{*}$ such that $f_{\alpha \mid X_{0}}=f_{0}$ and $\left\|f_{\alpha}\right\|^{*}=\left\|f_{0}\right\|^{*}$.

\section{Conflict of Interests}

The authors declare that there is no conflict of interests regarding the publication of this paper.

\section{Acknowledgments}

The authors thank the reviewers for their valuable suggestions. They also acknowledge the support of the Spanish 
Ministry of Economy and Competitiveness under Grant MTM2012-37894-C02-01.

\section{References}

[1] T. Bag and S. K. Samanta, "Fuzzy bounded linear operators," Fuzzy Sets and Systems, vol. 151, no. 3, pp. 513-547, 2005.

[2] A. K. Katsaras, "Fuzzy topological vector spaces. II," Fuzzy Sets and Systems, vol. 12, no. 2, pp. 143-154, 1984.

[3] C. Felbin, "Finite-dimensional fuzzy normed linear space," Fuzzy Sets and Systems, vol. 48, no. 2, pp. 239-248, 1992.

[4] O. Kaleva and S. Seikkala, "On fuzzy metric spaces," Fuzzy Sets and Systems, vol. 12, no. 3, pp. 215-229, 1984.

[5] S. C. Cheng and J. N. Mordeson, "Fuzzy linear operators and fuzzy normed linear spaces," Bulletin of the Calcutta Mathematical Society, vol. 86, no. 5, pp. 429-436, 1994.

[6] I. Kramosil and J. Michálek, "Fuzzy metrics and statistical metric spaces," Kybernetika, vol. 11, no. 5, pp. 336-344, 1975.

[7] T. Bag and S. K. Samanta, "Finite dimensional fuzzy normed linear spaces," Journal of Fuzzy Mathematics, vol. 11, no. 3, pp. 687-705, 2003.

[8] B. Schweizer and A. Sklar, "Statistical metric spaces," Pacific Journal of Mathematics, vol. 10, pp. 313-334, 1960.

[9] C. Alegre and S. Romaguera, "Characterizations of metrizable topological vector spaces and their asymmetric generalizations in terms of fuzzy (quasi-)norms," Fuzzy Sets and Systems, vol. 161, no. 16, pp. 2181-2192, 2010.

[10] V. Radu, "On the relationship between locally $(K)$-convex spaces and random normed spaces over valued fields," in Seminarul de Teoria Probabilitatilor STPA, vol. 37, West University of Timisoara, 1978.

[11] A. N. Serstnev, "On the notion on random normed space," Doklady Akademii Nauk SSSR, vol. 49, pp. 280-285, 1963.

[12] R. Meise and D. Vogt, Introduction to Functional Analysis, Oxford University Press, New York, NY, USA, 1967.

[13] C. D. Aliprantis and K. C. Border, Infinite Dimensional Analysis, Springer, New York, NY, USA, 2006. 


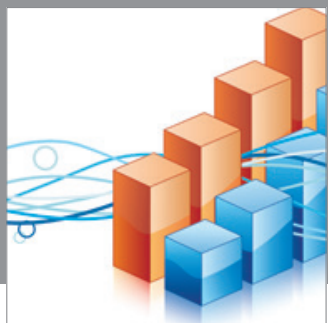

Advances in

Operations Research

mansans

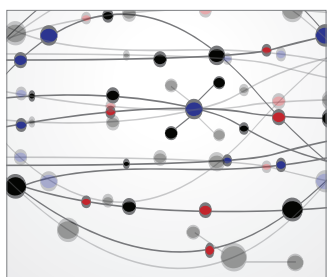

The Scientific World Journal
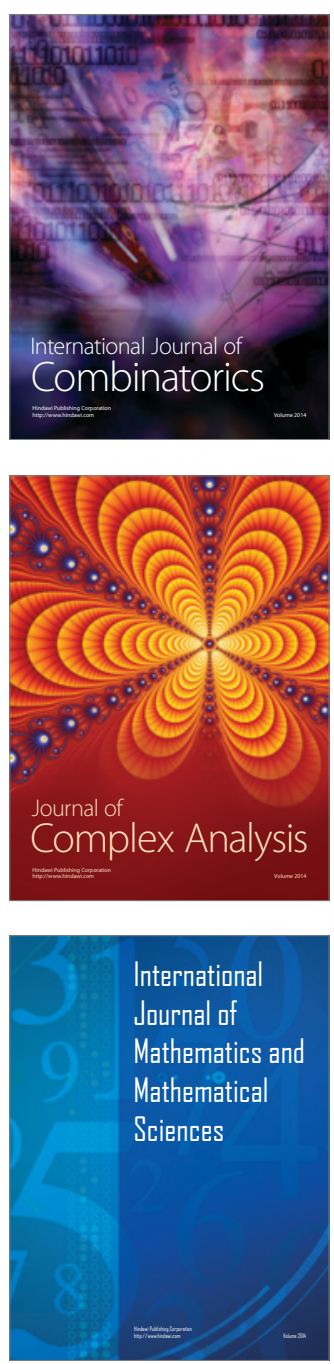
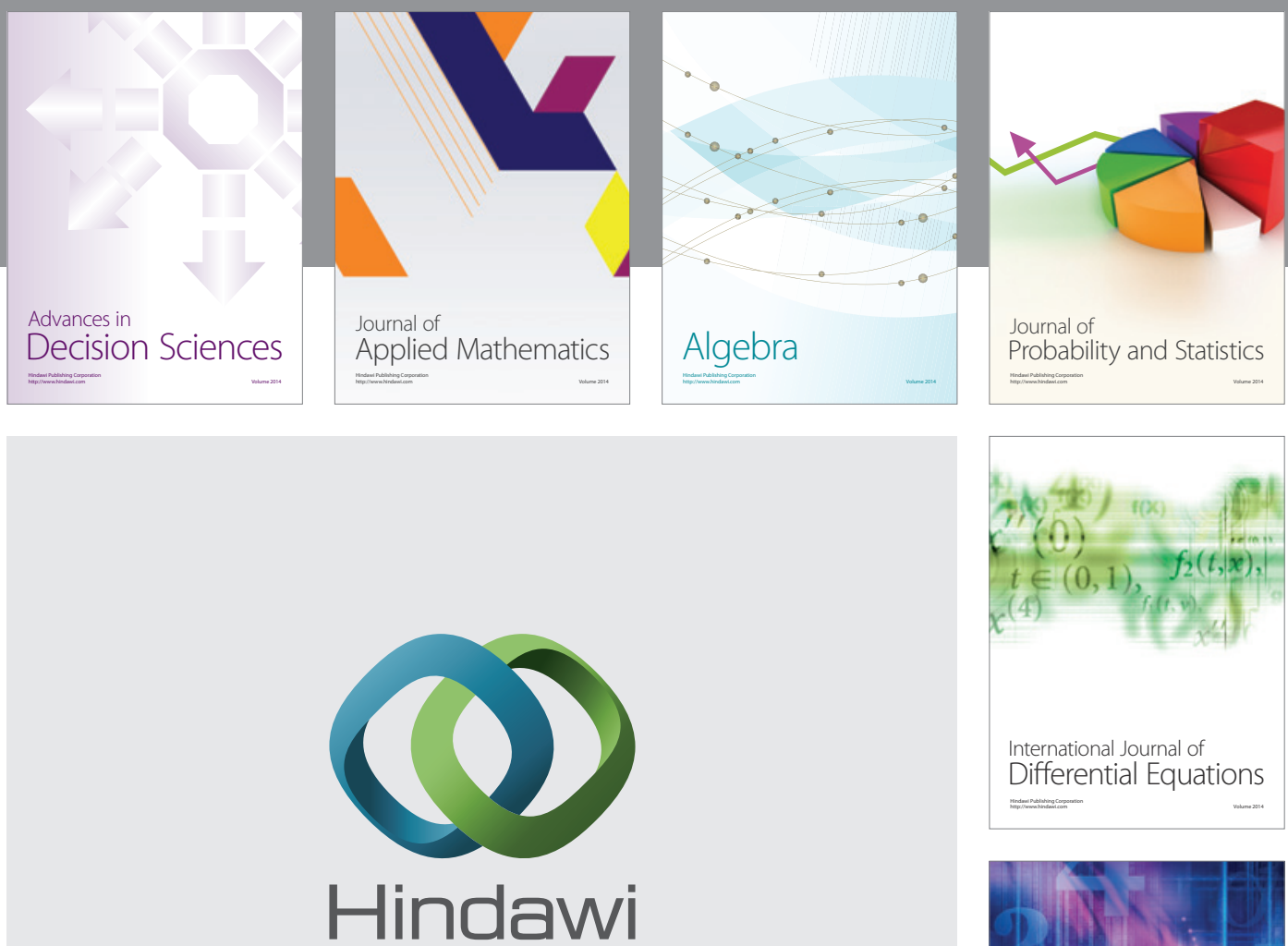

Submit your manuscripts at http://www.hindawi.com
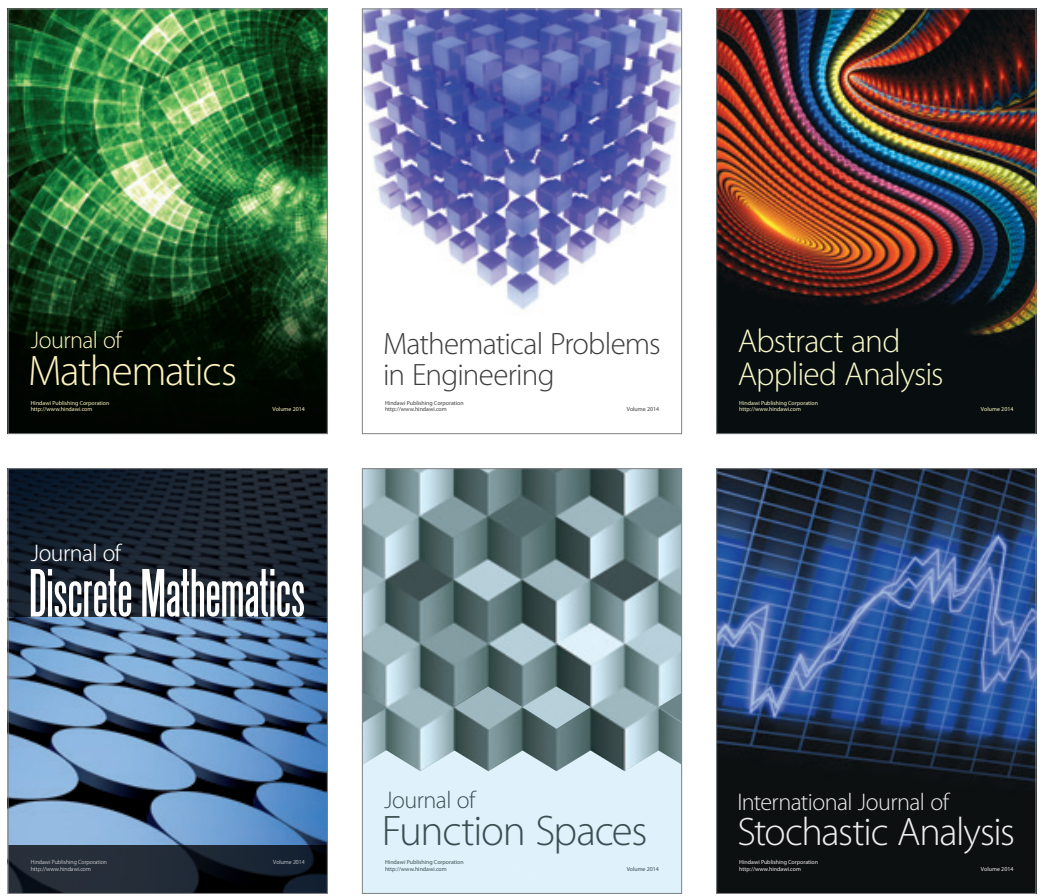

Journal of

Function Spaces

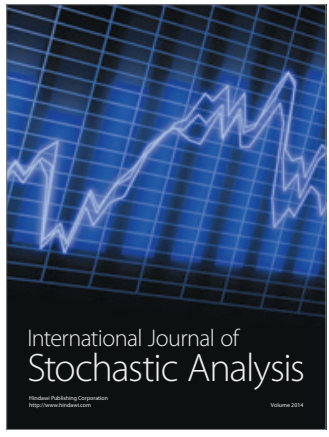

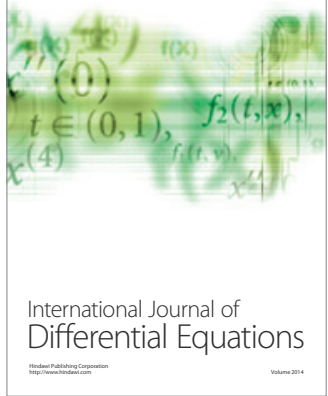
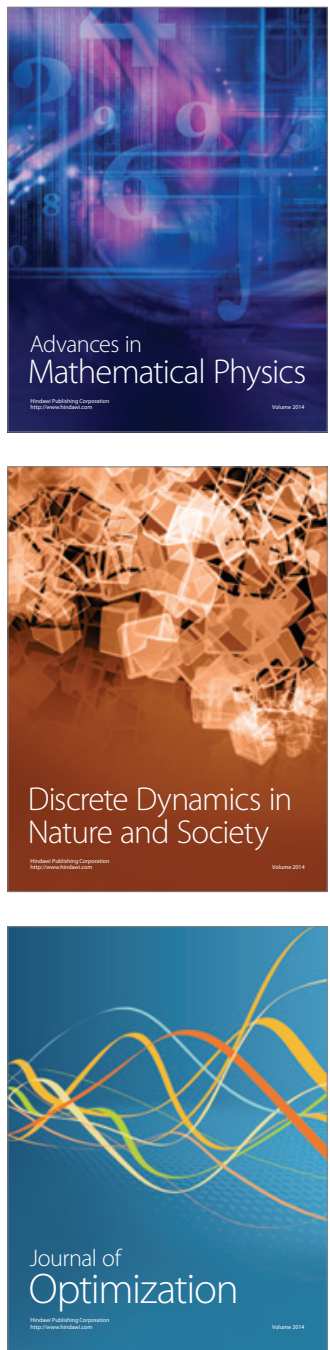